\title{
Visible-light-promoted nitrone synthesis from nitrosoarenes under catalyst- and additive-free conditions
}

\author{
Bao-Gui Cai ${ }^{1} \cdot$ Lin Li $^{1} \cdot$ Guo-Yong Xu${ }^{4} \cdot$ Wen-Jing Xiao ${ }^{3}$ Jun Xuan ${ }^{1,2}$
}

Received: 15 April 2021 / Accepted: 27 May 2021 / Published online: 11 June 2021

(c) The Author(s), under exclusive licence to European Photochemistry Association, European Society for Photobiology 2021

\begin{abstract}
A green and sustainable nitrone formation reaction via visible-light-promoted reaction of aryl diazoacetates with nitrosoarenes is described. This protocol exhibits good functional group tolerance and broad substrate scope for both aryl diazoacetates with nitrosoarenes. Comparing the reported methods for the synthesis of nitrones from nitrosoarenes, the reaction described herein occurs under sole visible-light irradiation without the need of any catalysts and additives.
\end{abstract}

\section{Graphic abstract}

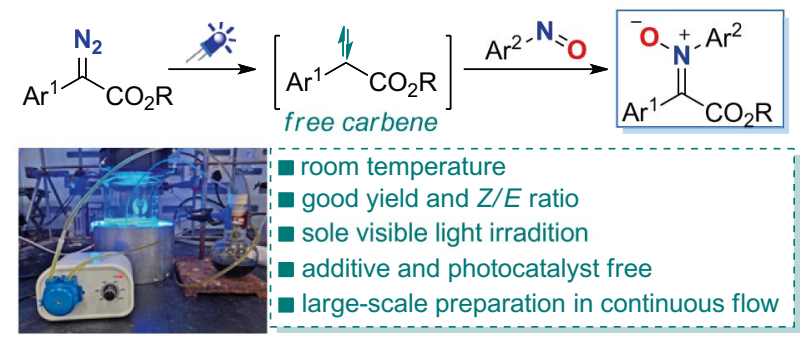

Keywords Nitrones $\cdot$ Visible light $\cdot$ Carbene $\cdot$ Nitrosoarenes

Guo-Yong Xu

gyxu@mail.ustc.edu.cn

$\triangle$ Wen-Jing Xiao

wxiao@mail.ccnu.edu.cn

$\triangle$ Jun Xuan

xuanjun@ahu.edu.cn

1 Anhui Province Key Laboratory of Chemistry for Inorganic/Organic Hybrid Functionalized Materials and Key Laboratory of Functional Inorganic Materials of Anhui Province, College of Chemistry \& Chemical Engineering, Anhui University, Hefei, Anhui 230601, People's Republic of China

2 Key Laboratory of Structure and Functional Regulation of Hybrid Materials (Anhui University), Ministry of Education, Hefei 230601, People's Republic of China

3 Key Laboratory of Pesticide \& Chemical Biology, Ministry of Education, College of Chemistry, Central China Normal University, 152 Luoyu Road, Wuhan, Hubei 430079, People's Republic of China

4 Institute of Physical Science and Information Technology, Anhui University, Hefei, Anhui 230601, People's Republic of China

\section{Introduction}

Nitrones are important synthetic building blocks in synthetic organic chemistry [1-4]. Generally, nitrones always used 1,3-dipoles to participate cycloaddition reactions to construct biologically important heterocyclic compounds [5-9]. In addition, nitrones can act as electrophiles to react with various nucleophiles to form $N, N$-disubstituted hydroxylamines [10-12] or as radical acceptors to undergo radical addition to create new chemical bonds [13, 14]. Under transition metal catalytic conditions, they can also be used as directing groups to realize $\mathrm{sp}^{2} \mathrm{C}-\mathrm{H}$ activation reactions $[15,16]$. In addition, many others useful reaction types of nitrones are developed in the past several years [17-21].

Due to their wide synthetic applications, many methods towards the facile synthesis of nitrones have been developed (Scheme 1). Condensation of $N$-monosubstituted hydroxylamines with aldehydes or ketones is an effective method for the preparation of nitrones [1-4, 22-24]. However, those 


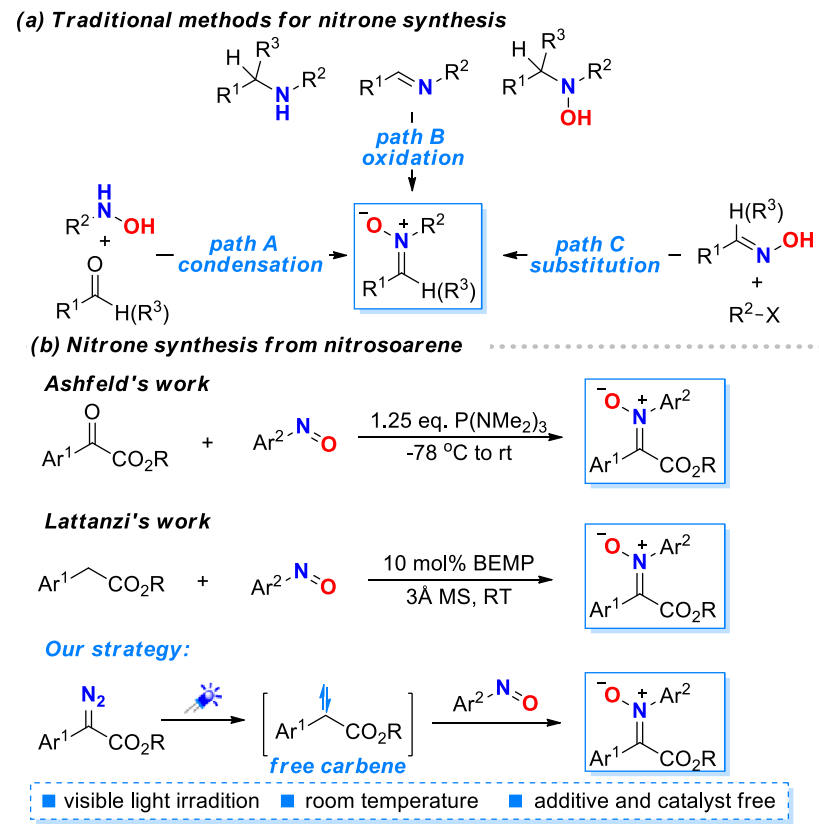

Scheme 1 Methods for the synthesis of nitrones

processes always require dehydrating reagents or perform at high temperature in a microwave apparatus (Scheme 1a, path A). Oxidation of secondary amines, imines or $N, N$ disubstituted hydroxylamines is another elegant route to form nitrones which often needs the consumption of stoichiometric oxidants (Scheme 1a, path B) [25-27] . In addition, the reaction to oximes with various electrophiles in the presence of metal catalysts to obtain nitrones has also attracted much attention (Scheme 1a, path C) [28]. Compared with those well-developed methods, nitrones synthesis from commercially available raw material nitrosobenzene is relatively undeveloped [29, 30]. In 2014, Ashfeld and their co-workers reported a hexamethylphosphorous $\left(\mathrm{P}\left(\mathrm{NMe}_{2}\right)_{3}\right)$ triamide-mediated addition of 1,2-dicarbonyls to nitroso compounds to construct arylnitrones. Using this umpolung strategy, the corresponding arylnitrones can be obtained in moderate to good yield. However, stoichiometric amounts of $\mathrm{P}\left(\mathrm{NMe}_{2}\right)_{3}$ are needed (Scheme 1b) [29]. Recently, Lattanzi and Mazzanti et al. developed the constructed arylnitrones from aryl acetates and nitrosoarenes in the presence of catalytic amount of 2-tert-butylimino-2-diethylamino1,3-dimethylperhydro-,3,2-diazaphosphorine (BEMP) as catalyst (Scheme 1b) [30]. Moreover, other types of nitrone construction from nitrosoarenes using transition metal- and dienamine catalytic strategy have also be realized [31-38]. Despite these elegant progresses, to the best of our knowledge, catalyst and additive-free synthesis of nitrones from nitrosoarenes has not yet been discovered. Our group recently found that photo-generated carbene species from diazoalkanes can be efficiently trapped by nitrosoarenes under sole blue LED irradiation [39], thus providing a novel and efficient route to amides under benign reaction conditions [40-68]. Control experiments revealed that nitrones were generated as the key intermediates which rapidly rearranged to the corresponding amides under blue LED irradiation. Based on these findings and our ongoing research interests on the development of visible-light-promoted fine chemical transformations [69-75], we hypothesized whether the nitrone intermediates can be isolated through optimizing the reaction paraments. Comparing the reported methods for the construction of nitrones from nitrosoarenes, the reaction described herein occurred using visible light as green energy source without the need of any catalysts and additives (Scheme 1b).

\section{Experimental}

\subsection{General information}

All reactions involving air- or moisture-sensitive reagents or intermediates were carried out in pre-heated glassware under an argon atmosphere using standard Schlenk techniques. All solvents and reagents were purified according to standard procedures or were used as received from chemical suppliers. The starting materials were synthesized according to literature procedures. The light employed in this work was bought from GeAo Chemical: model H106062, 24 W blue LEDs. Analytical thin layer chromatography was performed using Qingdao Puke Parting Materials Co. silica gel plates (Silica gel 60 F254). Visualization was by ultraviolet fluorescence $(\lambda=254 \mathrm{~nm})$ and/or staining with phosphomolybdic acid or potassium permanganate $\left(\mathrm{KMnO}_{4}\right)$. Flash column chromatography was performed using 200-300 mesh silica gel. ${ }^{1} \mathrm{H}$ NMR and ${ }^{13} \mathrm{C}$ NMR spectra were recorded on a JEOL JNM ECZ400R at $300 \mathrm{~K}$. Spectra were calibrated relative to solvent's residual proton and carbon chemical shift: $\mathrm{CHCl}_{3}\left(\delta=7.26\right.$ for ${ }^{1} \mathrm{H}$ NMR and $\delta=77.0$ for ${ }^{13} \mathrm{C}$ NMR). Data are reported as follows: chemical shift $\delta / \mathrm{ppm}$, integration $\left({ }^{1} \mathrm{H}\right.$ only), multiplicity $(\mathrm{s}=$ singlet, $\mathrm{d}=$ doublet, $\mathrm{t}=$ triplet, $\mathrm{q}=$ quartet, $\mathrm{dd}=$ doublet of doublets, $\mathrm{m}=$ multiplet or combinations thereof; ${ }^{13} \mathrm{C}$ signals are singlets unless otherwise stated), coupling constants $J$ in $\mathrm{Hz}$, assignment. Mass spectra were recorded on a Finnigan MAT 4200S, a Bruker Daltonics Micro Tof, a Waters-Micromass Quatro LCZ (ESI); peaks are given in $\mathrm{m} / \mathrm{z}$ (\% of basis peak). Melting points were determined by Stuart SMP10 and are uncorrected.

\subsection{General procedure for the synthesis of 3}

To a $10 \mathrm{~mL}$ Schlenk flask equipped with a magnetic stir bar was added $1(0.2 \mathrm{mmol}), 2(0.1 \mathrm{mmol})$, dry THF $(1.0 \mathrm{~mL})$. 
The resulting mixture was degassed via 'freeze-pump-thaw' procedure ( 3 times). After the solution was stirred at a distance of $\sim 3 \mathrm{~cm}$ from a $24 \mathrm{~W}$ blue LED at room temperature for $4 \mathrm{~h}$. The solvent was removed by vacuum and the crude product was purified by flash chromatography on silica gel silica: 200-300; eluant: petroleum ether/ethyl acetate (20:1-5:1) to provide pure product.

\section{Results and discussion}

At the beginning, 2-diazo-2-phenylacetate 1a with nitrosobenzene 2a was selected as model substrates to optimize the reaction conditions (Table 1 ). It was found that the reaction time had significant influence on the product ratio. Using DCM as solvent, the corresponding nitrone 3aa can be isolated in $46 \%$ yield under blue light irradiation in $4 \mathrm{~h}$ (Table 1, entry 1). Note that, 3aa will rearrange to amide by prolonging the reaction time [39]. To further improve the reaction efficiency, other reaction paraments were systematically screened (Table 1 , entries $2-5$ ). It was found that most of the solvents were suitable for the formation of desired nitrone 3aa, and THF was determined as the best reaction medium (Table 1, entry 3). Control experiments were also performed, and no reaction occurred in the absence of the light irradiation (Table 1, entry 6). It should be pointed out that $23 \%$ yield of the desired nitrone can be obtained when the reaction temperature raised to $50{ }^{\circ} \mathrm{C}$ in dark conditions (Table 1, entry 7).

Under the optimized reaction conditions, we first investigated the substrate scope for the aryldiazoacetate components (Table 2). In general, most of the reactions proceeded smoothly to afford the desired nitrones in moderate
Table 2 Scope of aryldiazoacetates

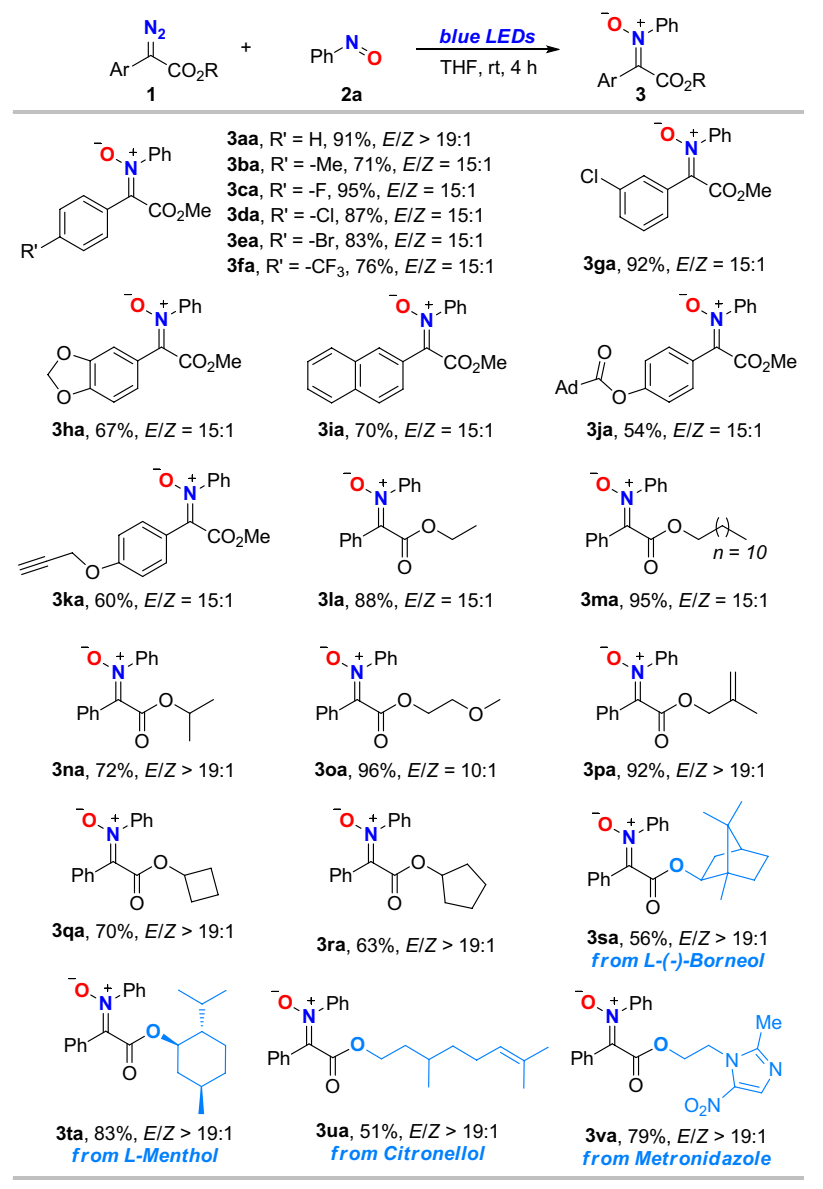

${ }^{a}$ Reaction performed with $1(0.2 \mathrm{mmol}), \mathbf{2 a}(0.1 \mathrm{mmol})$ in dry THF $(1.0 \mathrm{~mL})$ at $\mathrm{rt}$ under irradiation with $24 \mathrm{~W}$ blue LEDs for $4 \mathrm{~h}$. ${ }^{b}$ isolated yield.

Reaction performed with $1(0.2 \mathrm{mmol}), \mathbf{2 a}(0.1 \mathrm{mmol})$ in dry THF $(1.0 \mathrm{~mL})$ at $\mathrm{rt}$ under irradiation with $24 \mathrm{~W}$ blue LEDs for $4 \mathrm{~h}$. Isolated yield

to good yields and high $E / Z$ ratio. Both electron-rich ( $-\mathrm{Me})$ and electron-deficient substituents $\left(-\mathrm{F},-\mathrm{Cl},-\mathrm{Br},-\mathrm{CF}_{3}\right)$ at para or meta-positions of the aromatic ring turned out to be suitable, providing the corresponding nitrones 3aa-3ga in excellent yields (71-95\%) and good diastereoselectivity. To our delight, reaction with aryldiazoacetates bearing benzo $[d][1,3]$ dioxole (1h), naphthyl (1i) also proceeded well. Note that nitrone containing adamantane formic acid (3ja) and replacement of the methyl group with other alkyl propargyl (3ka) attached to the aryl group can be obtained in $54 \%$ and $60 \%$, respectively. In addition, groups (11-n), cyclic alkyl groups (1q and 1r) or the substituents containing sensitive functional groups, e.g., ether (10), alkene (1p), all successfully yielded the corresponding nitrones in good to excellent yields (63-96\%) and high $E / Z$ ratio. We also introduced some natural products or drug molecules into the nitrone skeletons and applied them under the optimal
Reaction conditions: 1a $(0.2 \mathrm{mmol}), \mathbf{2 a}(0.1 \mathrm{mmol})$ in dry solvent $(1.0 \mathrm{~mL})$ at $\mathrm{rt}$ under irradiation with $24 \mathrm{~W}$ blue LEDs for $4 \mathrm{~h}$

${ }^{\text {a }}$ Isolated yield. ${ }^{c}$ in dark, at $50{ }^{\circ} \mathrm{C}$ 
reaction conditions. Pleasingly, natural isolates $L-(-)-B o r-$ neol (1 s), L-Menthol (1t), Citronellol (1u) were successfully converted into nitrones in moderate to high yields (51-83\%) and with $E / Z>19: 1$. Metronidazole also smoothly gave the drug-modified nitrone 3va with $79 \%$ yields.

Next, we examined the compatibility of different nitrosoarenes and the results is summarized in Table 3. Under the optimized reaction conditions, various halogen $(\mathbf{2 b}, \mathbf{2 c}$, 2g, 2i), alkyl (2e, 2h), alkoxyl (2k) and aryl (2f) substituted nitrosoarenes were all suitable for this reaction, providing the corresponding products in $52-95 \%$ yields with $10: 1$ E/Z ratio. To our delight, nitrosoarenes bearing ester (2d) and ketone (2j) fragments were well tolerated and afforded desired nitrones in $75 \%$ and $66 \%$ yields, respectively. Apart from phenylnitrosoarene, substrate $\mathbf{2 l}$ bearing 2-nitrosopyridine was amenable substrate, giving nitrone 3al in $80 \%$ yields with high diastereoselectivity. Note that, 2-methyl2-nitrosopropane 2 failed to give the final product, this might due to the steric of the ${ }^{t} \mathrm{Bu}$ group. The successful introduction of Pterostilbene (2n) and Vitamin E (2o) fragment into final nitrone also revealed the advantage of this visible-lightinduced nitrone formation strategy.

With the aim at showing the utility of current methodology, we implemented a gram-scale synthesis under continuous flow reaction conditions (Scheme 2a). To our delight,

Table 3 Scope of nitrosoarenes

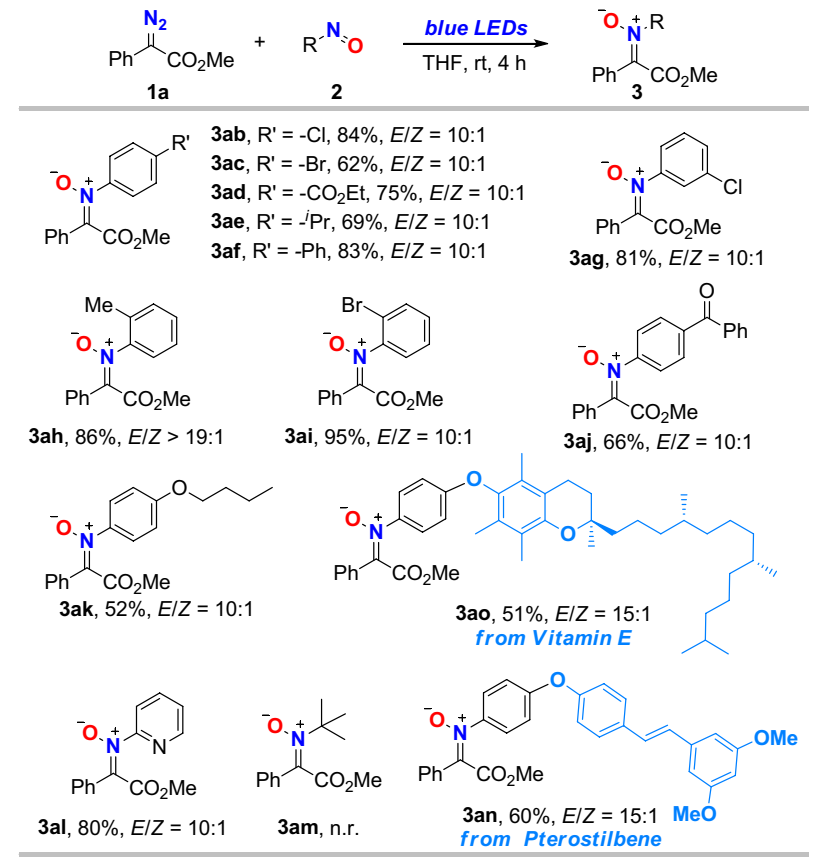

${ }^{a}$ Reaction performed with $1(0.2 \mathrm{mmol}), \mathbf{2 a}(0.1 \mathrm{mmol})$ in dry THF $(1.0 \mathrm{~mL})$ at $\mathrm{rt}$ under irradiation with $24 \mathrm{~W}$ blue LEDs for $4 \mathrm{~h}$. ${ }^{b}$ isolated yield.

Reaction performed with $\mathbf{1}(0.2 \mathrm{mmol}), \mathbf{2 a}(0.1 \mathrm{mmol})$ in dry THF $(1.0 \mathrm{~mL})$ at $\mathrm{rt}$ under irradiation with $24 \mathrm{~W}$ blue LEDs for $4 \mathrm{~h}$. Isolated yield nitrone 3aa can be obtained in $89 \%$ yield using phenyldiazoacetate 1a and nitrosobenzene $\mathbf{2 a}$ as substrates. Moreover, treatment of 3aa under reductive conditions with zinc powder and $\mathrm{NH}_{4} \mathrm{Cl}$ provided amino ester derivative 4 in $91 \%$ yields (Scheme 2b) [76].

Some preliminary control experiments were conducted to gain some insight into the mechanism. When 1.0 equivalent of radical scavenger TEMPO was added under standard conditions, 3aa still could be isolated in $92 \%$ yield. The result indicated that a radical pathway might be not involved in current reaction (Scheme 3a). It was reported that ethyl diazoacetate is an efficient carbene-trapping reagent under photochemical conditions [65]. When 1.0 equivalent of ethyl diazoacetate was added as a trapping reagent, the reaction provided nitrone 3aa in $74 \%$ yield, together with $11 \%$ yield of alkene $\mathbf{6}$, which suggested the carbene species formed during the reaction (Scheme $3 b$ ).

On the basis of above experimental results and literature report [51-68], a plausible reaction mechanism was proposed in Scheme 3. Initially, visible-light irradiation of aryldiazoacetate resulted in $\mathrm{N}_{2}$ gas extrusion and generated the reactive carbene species. The carbene intermediate reacts

(a) Gram-scale synthesis in continuous flow

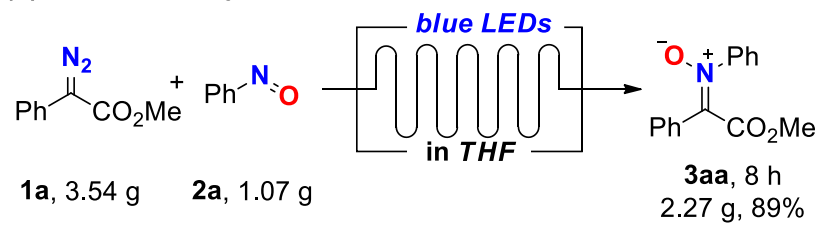

(b) Reduction of $3 a a$

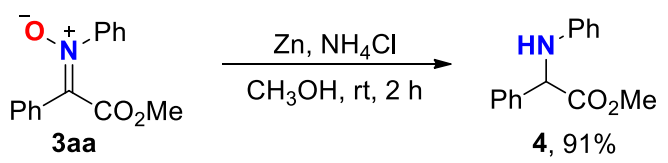

Scheme 2 Synthetic applications
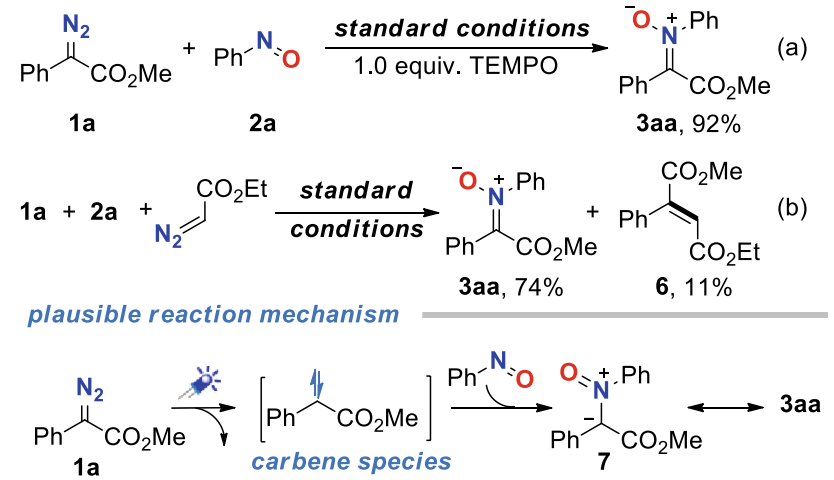

Scheme 3 Mechanism studies 
with a nitrosoarene via its nucleophilic $\mathrm{N}$-center to give the zwitterion intermediate $\mathbf{7}$, which converts the more stable nitrone 3aa as the predominant resonance form.

\section{Conclusions}

In summary, we have developed a green and sustainable nitrone formation protocol via visible-light-promoted reaction of aryl diazoacetates with nitrosoarenes. Comparing the reported methods for the construction of nitrones from nitrosoarenes, the reaction described herein occurred under sole visible-light irradiation without the need of any catalysts and additives. The reaction showed good substrate scope, excellent functional group tolerance for both aryl diazoacetate and nitrosoarene components. Moreover, the success synthesis of natural product- or drug moleculemodified nitrones, large-scale preparation in continuous flow conditions further render the method attractive and valuable.

Supplementary Information The online version contains supplementary material available at https://doi.org/10.1007/s43630-021-00062-6.

Acknowledgements We are grateful to the National Natural Science Foundation of China (Nos. 21971001, 21702001), the Natural Science Founda-tion of Anhui Province (No. 1808085MB47) and the Start-up Grant from Anhui University for financial support of this work.

\section{Declarations}

Conflict of interest The authors declare that they have no conflict of interest.

\section{References}

1. Jones, R. C. F., \& Martin, J. N. (2003). Synthetic applications of 1,3-dipolar cycloadditions. In A. Padwa \& W. H. Pearson (Eds.), Chemistry toward heterocycles and natural products. Hoboken: Wiley.

2. Torssell, K. B. G. (1988). In H. Feuer (Ed.), Nitrile oxides nitrones and ntronates in organic synthesis. Weinheim: Wiley VCH.

3. Tufariello, J. J. (1984). In A. Padwa (Ed.), 1,3-Dipolar cycloaddition chemistry. (Vol. 2). New York: Wiley.

4. Murahashi, S.-I., \& Imada, Y. (2019). Synthesis and transformations of nitrones for organic synthesis. Chemical Reviews. https:// doi.org/10.1021/acs.chemrev.8b00476

5. Gothelf, K. V., \& Jørgensen, K. A. (1998). Asymmetric 1,3-dipolar cycloaddition reactions. Chemical Reviews. https://doi.org/10. 1021/cr970324e

6. Stanley, L. M., \& Sibi, M. P. (2008). Enantioselective coppercatalyzed 1,3-dipolar cycloadditions. Chemical Reviews. https:// doi.org/10.1021/cr078371m

7. Hashimoto, T., \& Maruoka, K. (2015). Recent advances of catalytic asymmetric 1,3-dipolar cycloadditions. Chemical Reviews. https://doi.org/10.1021/cr5007182

8. Brandi, A., Cardona, F., Cicchi, S., Cordero, F. M., \& Goti, A. (2017). [3 + 2] dipolar cycloadditions of cyclic nitrones with alkenes. Organic Reactions. https://doi.org/10.1002/0471264180. or094.01

9. Xuan, J., Cheng, X., \& Cao, X. (2017). [3+3] Cycloaddition of in situ formed azaoxyallyl cations with nitrones: Synthesis of 1,2,4-oxadiazinan-5-one derivatives. ChemistrySelect. https:// doi.org/10.1002/slct.201700826

10. Merino, P., Franco, S., Merchan, F. L., \& Tejero, T. (2000). Nucleophilic additions to chiral nitrones: New approaches to nitrogenated compounds. Synlett. https://doi.org/10.1055/s-2000-6555

11. Lombardo, M., \& Trombini, C. (2000). Nucleophilic additions to nitrones. Synthesis. https://doi.org/10.1055/s-2000-6269

12. Revuelta, J., Cicchi, S., Goti, A., \& Brandi, A. (2007). Enantiopure cyclic nitrones: A useful class of building blocks for asymmetric syntheses. Synthesis. https://doi.org/10.1055/s-2007-965914

13. Ueda, M., Miyabe, H., Teramachi, M., Miyata, O., \& Naito, T. (2003). Novel intermolecular carbon radical addition to a nitrone: Asymmetric synthesis of $\alpha$-amino acids. Chemical Communications. https://doi.org/10.1039/B211570K

14. Ueda, M., Miyabe, H., Teramachi, M., Miyata, O., \& Naito, T. (2005). Diastereoselective intermolecular radical addition to nitrones. The Journal of Organic Chemistry. https://doi.org/10. 1021/jo050603z

15. Xie, F., Qi, Z., Yu, S., \& Li, X. (2014). Rh(III)- and Ir(III)-Catalyzed C-H alkynylation of arenes under chelation assistance. Journal of the American Chemical Society. https://doi.org/10.1021/ ja501910e

16. Deng, H., Li, H., Zhang, W., \& Wang, L. (2017). Rh ${ }^{\mathrm{III}}$-Catalyzed site-selective amidation with nitrone as a traceless directing group: An approach to functionalized arylaldehydes. Chemical Communications. https://doi.org/10.1039/C7CC05297A

17. Bøgevig, A., Gothelf, K. V., \& Jørgensen, K. A. (2002). Nucleophilic addition of nitrones to ketones: Development of a new catalytic asymmetric nitrone-aldol reaction. Chemistry A European Journal. https://doi.org/10.1002/1521-3765(20021216)8:24\% 3c5652::AID-CHEM5652\%3e3.0.CO;2-J

18. Berini, C., Minassian, F., Pelloux-Léon, N., Denis, J.-N., Vallée, Y., \& Philouze, C. (2008). Efficient stereoselective nucleophilic addition of pyrroles to chiral nitrones. Organic \& Biomolecular Chemistry. https://doi.org/10.1039/B802997K

19. Richmond, E., Duguet, N., Slawin, A. M. Z., Lébl, T., \& Smith, A. D. (2012). Asymmetric pericyclic cascade approach to spirocyclic oxindoles. Organic Letters. https://doi.org/10.1021/ol300982f

20. Pace, W. H., Mo, D.-L., Reidl, T. W., Wink, D. J., \& Anderson, L. L. (2016). Catalytic asymmetric synthesis of dihydropyrido[1,2-a] indoles from nitrones and allenoates. Angewandte International Edition Chemie. https://doi.org/10.1002/anie.201602568

21. Son, J., Kim, K. H., Mo, D. L., Wink, D. J., \& Anderson, L. L. (2017). Single-step modular synthesis of unsaturated morpholine $\mathrm{N}$-oxides and their cycloaddition reactions. Angewandte Chemie International Edition. https://doi.org/10.1002/anie.201611791

22. LeBel, N. A., \& Banucci, E. G. (1971). Intramolecular nitroneolefin cycloadditions. Stereochemistry of hexahydro-2,1-benzisoxazoline formation. The Journal of Organic Chemistry. https://doi.org/10.1021/jo00816a013

23. Andrade, M. M., Barros, M. T., \& Pinto, R. C. (2008). Exploiting microwave-assisted neat procedures: Synthesis of $\mathrm{N}$-aryl and $\mathrm{N}$-alkylnitrones and their cycloaddition en route for isoxazolidines. Tetrahedron. https://doi.org/10.1016/j.tet.2008.08.101

24. Pfeiffer, J. Y., \& Beauchemin, A. M. (2009). Simple reaction conditions for the formation of ketonitrones from ketones and hydroxylamines. The Journal of Organic Chemistry. https://doi. org/10.1021/jo901653d

25. Soldaini, G., Cardona, F., \& Goti, A. (2007). Catalytic oxidation of imines based on methyltrioxorhenium/urea hydrogen peroxide: A mild and easy chemo- and regioselective entry to nitrones. Organic Letters. https://doi.org/10.1021/o1062862w 
26. Colladon, M., Scarso, A., \& Strukul, G. (2008). Mild catalytic oxidation of secondary and tertiary amines to nitrones and $\mathrm{N}$-oxides with $\mathrm{H}_{2} \mathrm{O}_{2}$ mediated by $\mathrm{Pt}(\mathrm{II})$ catalysts. Green Chemistry. https://doi.org/10.1039/B805404E

27. Matassini, C., Parmeggiani, C., Cardona, F. \& Goti, A. (2015). Oxidation of N,N-disubstituted hydroxylamines to nitrones with hypervalent iodine reagents. Organic Letters. https://doi.org/10. 1021/acs.orglett.5b02029.

28. Reidl, T. W., Son, J., Wink, D. J., \& Anderson, L. L. (2017). Facile synthesis of azetidine nitrones and diastereoselective conversion into densely substituted azetidines. Angewandte Chemie International Edition. https://doi.org/10.1002/anie. 201705681

29. Chavannavar, A. P., Oliver, A. G., \& Ashfeld, B. L. (2014). An umpolung approach toward $\mathrm{N}$-aryl nitrone construction: A phosphine-mediated addition of 1,2-dicarbonyls to nitroso electrophiles. Chemical Communications. https://doi.org/10.1039/ C4CC05044D

30. Volpe, C., Meninno, S., Roselli, A., Mancinelli, M., Mazzanti, A., \& Lattanzi, A. (2020). Nitrone/imine selectivity switch in basecatalysed reaction of aryl acetic acid esters with nitrosoarenes: Joint experimental and computational study. Advanced Synthesis \& Catalysis. https://doi.org/10.1002/adsc.202000855

31. Reddy, A. R., Guo, Z., Siu, F.-M., Lok, C.-N., Liu, F., Yeung, K.-C., Zhou, C.-Y., \& Che, C.-M. (2012). Diastereoselective ruthenium porphyrin-catalyzed tandem nitrone formation/1,3dipolar cycloaddition for isoxazolidines. Synthesis, in silico docking study and in vitro biological activities. Organic \& Biomolecular Chemistry. https://doi.org/10.1039/C2OB26518D

32. Reddy, A. R., Zhou, C.-Y., \& Che, C.-M. (2014). Ruthenium porphyrin catalyzed three-component reaction of diazo compounds, nitrosoarenes, and alkynes: An efficient approach to multifunctionalized aziridines. Organic Letters. https://doi.org/10.1021/ ol4035098

33. Singh, R. R., \& Liu, R.-S. (2014). Gold-catalyzed 1,2-iminonitronation of electron-deficient alkynes with nitrosoarenes to afford $\alpha$-imidoyl nitrones. Chemical Communications. https://doi.org/ 10.1039/C4CC06962E

34. Pagar, V. V., \& Liu, R.-S. (2015). Gold-catalyzed cycloaddition reactions of ethyl diazoacetate, nitrosoarenes, and vinyldiazo carbonyl compounds: Synthesis of isoxazolidine and benzo[b] azepine derivatives. Angewandte Chemie International Edition. https://doi.org/10.1002/anie.201500340

35. Wu, M.-Y., He, W.-W., Liu, X.-Y., \& Tan, B. (2015). Asymmetric construction of spirooxindoles by organocatalytic multicomponent reactions using diazooxindoles. Angewandte Chemie International Edition. https://doi.org/10.1002/anie.201504640

36. Fraboni, A. J., \& Brenner-Moyer, S. E. (2016). Dienaminecatalyzed nitrone formation via redox reaction. Organic Letters. https://doi.org/10.1021/acs.orglett.6b00770

37. Li, X., Feng, T., Li, D., Chang, H., Gao, W., \& Wei, W. (2019). KOAc-Catalyzed one-pot three-component 1,3-dipolar cycloaddition of $\alpha$-diazo compounds, nitrosoarenes, and alkenes: An approach to functionalized isoxazolidines. The Journal of Organic Chemistry. https://doi.org/10.1021/acs.joc.9b00299

38. Wróbel, Z., \& Kwasr, A. (2007). 2-Nitroso-N-arylanilines: Products of acid-promoted transformation of ${ }^{\sigma} \mathrm{H}$ adducts of arylamines and nitroarenes. Synlett. https://doi.org/10.1055/s-2007-982534

39. Cai, B.-G., Luo, S.-S., Li, L., Li, L., Xuan, J., \& Xiao, W.-J. (2020). Visible light-promoted amide bond formation via one-pot nitrone in situ formation/rearrangement cascade. CCS Chemistry. https://doi.org/10.31635/ccschem.020.202000588

40. Narayanam, J. M. R., \& Stephenson, C. R. J. (2011). Visible light photoredox catalysis: Applications in organic synthesis. Chemical Society Reviews. https://doi.org/10.1039/B913880N
41. Xuan, J., \& Xiao, W.-J. (2012). Visible-light photoredox catalysis. Angewandte Chemie International Edition. https://doi.org/10. 1002/anie.201200223

42. Xi, Y., Yi, H., \& Lei, A. (2013). Synthetic applications of photoredox catalysis with visible light. Organic \& Biomolecular Chemistry. https://doi.org/10.1039/C3OB40137E

43. Prier, C. K., Rankic, D. A., \& MacMillan, D. W. C. (2013). Visible light photoredox catalysis with transition metal complexes: Applications in organic synthesis. Chemical Reviews. https://doi. org/10.1021/cr300503r

44. Hopkinson, M. N., Sahoo, B., Li, J.-L., \& Glorius, F. (2014). Dual catalysis sees the light: Combining photoredox with organo-, acid, and transition-metal catalysis. Chemistry A European Journal. https://doi.org/10.1002/chem.201304823

45. Marzo, L., Pagire, S., Reiser, O., \& König, B. (2018). Visible-light photocatalysis: Does it make a difference in organic synthesis? Angewandte Chemie International Edition. https://doi.org/10. 1002/anie.201709766

46. Chen, Y., Lu, L.-Q., Yu, D.-G., Zhu, C.-J., \& Xiao, W.-J. (2019). Visible light-driven organic photochemical synthesis in china. Science China Chemistry. https://doi.org/10.1007/ s11426-018-9399-2

47. Cai, B.-G., Xuan, J., \& Xiao, W.-J. (2019). Visible light-mediated C-P bond formation reactions. Science Bulletin. https://doi.org/10. 1016/j.scib.2019.02.002

48. Yu, X.-Y., Zhao, Q.-Q., Chen, J., Xiao, W.-J., \& Chen, J.-R. (2020). When light meets nitrogen-centered radicals: From reagents to catalysts. Accounts of Chemical Research. https://doi.org/ 10.1021/acs.accounts.0c00090

49. Sun, K., Lv, Q.-Y., Chen, X.-L., Qu, L.-B., \& Yu, B. (2021). Recent advances in visible-light-mediated organic transformations in water. Green Chemistry. https://doi.org/10.1039/D0GC03447A

50. Sun, K., Lv, Q.-Y., Lin, Y.-W., Yu, B., \& He, W.-M. (2021). Nitriles as radical acceptors in radical cascade reactions. Organic Chemistry Frontiers. https://doi.org/10.1039/D0QO01058H

51. Ciszewski, L. W., Rybicka-Jasinska, K., \& Gryko, D. (2019). Recent developments in photochemical reactions of diazo compounds. Organic \& Biomolecular Chemistry. https://doi.org/10. 1039/C8OB02703J

52. Yang, Z., Stivanin, M. L., Jurberg, I. D., \& Koenigs, R. M. (2020). Visible light-promoted reactions with diazo compounds: A mild and practical strategy towards free carbene intermediates. Chemical Society Reviews. https://doi.org/10.1039/D0CS00224K

53. Empel, C., \& Koenigs, R. M. (2019). Sustainable carbene transfer reactions with iron and light. Synlett. https://doi.org/10.1055/s0037-1611874

54. Jana, S., Guo, Y., \& Koenigs, R. M. (2021). Recent perspectives on rearrangement reactions of ylides via carbene transfer reactions. Chemistry A European Journal. https://doi.org/10.1002/ chem. 202002556

55. Jurberg, I., \& Davies, H. M. L. (2018). Blue light-promoted photolysis of aryldiazoacetates. Chemical Science. https://doi.org/10. 1039/C8SC01165F

56. Hommelsheim, R., Guo, Y., Yang, Z., Empel, C., \& Koenigs, R. M. (2019). Blue-light-induced carbene-transfer reactions of diazoalkanes. Angewandte Chemie International Edition. https:// doi.org/10.1002/anie.201811991

57. Jana, S., Yang, Z., Li, F., Empel, C., Ho, J., \& Koenigs, R. M. (2020). Photoinduced proton-transfer reactions for mild O-H functionalization of unreactive alcohols. Angewandte Chemie International Edition. https://doi.org/10.1002/anie.201915161

58. Jana, S., Yang, Z., Pei, C., Xu, X., \& Koenigs, R. M. (2019). Photochemical ring expansion reactions: Synthesis of tetrahydrofuran derivatives and mechanism studies. Chemical Science. https://doi. org/10.1039/C9SC04069B 
59. Guo, Y., Nguyen, T. V., \& Koenigs, R. M. (2019). Norcaradiene synthesis via visible-light-mediated cyclopropanation reactions of arenes. Organic Letters. https://doi.org/10.1021/acs.orglett.9b034 53

60. He, F., \& Koenigs, R. M. (2019). Visible light mediated, metalfree carbene transfer reactions of diazoalkanes with propargylic alcohols. Chemical Communications. https://doi.org/10.1039/ C9CC00927B

61. He, F., Li, F., \& Koenigs, R. M. (2020). Metal-free insertion reactions of silanes with aryldiazoacetates. The Journal of Organic Chemistry. https://doi.org/10.1021/acs.joc.9b02605

62. Empel, C., Patureau, F. W., \& Koenigs, R. M. (2019). Visible light induced metal-free carbene N-carbazolation. The Journal of Organic Chemistry. https://doi.org/10.1021/acs.joc.9b01753

63. Orlowska, K., Rybicka-Jasinska, K., Krajewski, P., \& Gryko, D. (2020). Photochemical doyle-kirmse reaction: A route to allenes. Organic Letters. https://doi.org/10.1021/acs.orglett.9b04560

64. Yang, J., Wang, J., Huang, H., Qin, G., Jiang, Y., \& Xiao, T. (2019). gem-Difluoroallylation of aryl diazoesters via catalystfree, blue-light-mediated formal doyle-kirmse reaction. Organic Letters. https://doi.org/10.1021/acs.orglett.9b00647

65. Xiao, T., Mei, M., He, Y., \& Zhou, L. (2018). Blue light-promoted cross-coupling of aryldiazoacetates and diazocarbonyl compounds. Chemical Communications. https://doi.org/10.1039/ C8CC04609C

66. Yang, Z., Guo, Y., \& Koenigs, R. M. (2018). Solvent-dependent, rhodium catalysed rearrangement reactions of sulfur ylides. Chemical Communications. https://doi.org/10.1039/C9CC03809D

67. Rybicka-Jasińska, K., Shan, W., Zawada, K., Kadish, K. M., \& Gryko, D. (2020). Porphyrins as photoredox catalysts: Experimental and theoretical studies. Journal of the American Chemical Society. https://doi.org/10.1021/jacs.6b09036

68. Cheng, R., Qi, C., Wang, L., Xiong, W., Liu, H., \& Jiang, H. (2020). Visible light-promoted synthesis of organic carbamates from carbon dioxide under catalyst- and additive-free conditions. Green Chemistry. https://doi.org/10.1039/D0GC00910E

69. Cai, B.-G., Chen, Z.-L., Xu, G.-Y., Xuan, J., \& Xiao, W.-J. (2019). $[3+2]$-cycloaddition of $2 \mathrm{H}$-azirines with nitrosoarenes: Visible-light-promoted synthesis of 2,5-dihydro-1,2,4-oxadiazoles. Organic Letters. https://doi.org/10.1021/acs.orglett.9b01416

70. Ge, Q.-Q., Qian, J.-S., \& Xuan, J. (2019). Electron donor-acceptor complex enabled eecarboxylative sulfonylation of cinnamic acids under visible-light irradiation. The Journal of Organic Chemistry. https://doi.org/10.1021/acs.joc.9b00552

71. He, X.-K., Cai, B.-G., Yang, Q.-Q., Wang, L., \& Xuan, J. (2019). Visible-light-promoted cascade radical cyclization: Synthesis of 1,4-diketones containing chroman-4-One Skeletons. Chemistry An Asian Journal. https://doi.org/10.1002/asia.201901078

72. He, X.-K., Lu, J., Zhang, A.-J., Zhang, Q.-Q., Xu, G.-Y., \& Xuan, J. (2020). BI-OAc-accelerated C3-H alkylation of quinoxalin$2(1 \mathrm{H})$-ones under visible-light irradiation. Organic Letters. https://doi.org/10.1021/acs.orglett.0c02080

73. Lu, J., He, X.-K., Cheng, X., Zhang, A.-J., Xu, G.-Y., \& Xuan, J. (2020). Photoredox catalyst free, visible light-promoted C3-H acylation of quinoxalin-2(1H)-ones in water. Advanced Synthesis \& Catalysis. https://doi.org/10.1002/adsc.202000116

74. Xuan, J., He, X.-K., \& Xiao, W.-J. (2020). Visible light-promoted ring-opening functionalization of three-membered carbo- and heterocycles. Chemical Society Reviews. https://doi.org/10.1039/ C9CS00523D

75. Ye, C., Cai, B.-G., Lu, J., Cheng, X., Li, L., Pan, Z.-W., \& Xuan, J. (2021). Visible-light-promoted polysubstituted olefins synthesis involving sulfur ylides as carbene trapping reagents. The Journal of Organic Chemistry. https://doi.org/10.1021/acs.joc.0c02500

76. Guo, W., Gu, J., \& Gu, Z. (2020). Catalytic asymmetric synthesis of atropisomeric nitrones: Versatile intermediate for axially chiral biaryls. Organic Letters. https://doi.org/10.1021/acs.orglett.0c028 30 